\section{Successive reversals of a visual social stimulus*}

\author{
CARL DANSON and THOMAS CREED† \\ California State College, Long Beach, Calif. 90801
}

A high rate and a near-zero rate of response of one monkey were successively reversed as the occasion for reinforcement and nonreinforcement of another monkey. Both rates of response were discriminated by the second monkey. The acquisition of the later discriminations in the sequence of reversals was more rapid than the earlier discriminations. These results indicate that a wide range of rates of response may serve as social stimuli and that the learning-set (learning-to-learning) phenomenon appears to be relevant to social as well as nonsocial episodes.

A recent experiment by the authors (Danson \& Creed, 1970) showed that the operant performance (chain pulling) of a stimulus monkey could develop visual discriminative control of the operant performance (barpressing) of an observer monkey. Experiment 2 of that investigation showed that the rate of chain pulling by the stimulus monkey was a crucial variable in the control of the $O$ 's performance. During an extinction test, a decrease in the rate of chain pulling yielded a decrease in the rate of barpressing. Control procedures eliminated temporal and nonsocial factors as responsible for the findings. The implication of the two experiments was that rate of response as a visual social stimulus functions similarly to other physical stimuli in terms of discrimination and generalization.

The present study was intended to test further the similarity of rate of response as a social stimulus to other physical stimuli by reversing the rate of response of the stimulus monkey that was the occasion for reinforcement $\left(\mathrm{S}^{\mathrm{D}}\right)$ for the $\mathrm{O}$ and the rate of the stimulus monkey that was correlated with nonreinforcement $\left(S^{\Delta}\right)$ for the $O$. In the earlier studies, a high rate of chain pulling served as $\mathrm{S}^{\mathrm{D}}$ and a near-zero rate (not chain pulling) served as $S^{\Delta}$. The experiment reported here was directed at answering two questions: (1) Would discriminative control of the $\mathrm{O}$ develop when a zero rate of the stimulus monkey served as the $S^{D}$ for the $\mathrm{O}$ and the high rate of the stimulus monkey was used as the $S^{\Delta}$ ? (2) And, if the reversal of the stimuli did gain effective control if the $O$ 's performance, would the successive reversal of the social $S^{D}$ and

* This research was supported in part by the Long Beach State College Research Foundation.

tThe authors wish to thank Lance Johnson who helped conduct the experimental sessions, Dave Stark for his technical assistance, and Dr. S. J. Weiss for his suggestions prior to the experiment.
$\mathrm{S}^{\Delta}$ produce a learning-set phenomenon (Kimble, 1961)?

Social stimuli have not frequently been employed in a series of discrimination problems, and in those cases where they have been (e.g., Darby \& Riopelle, 1959), the results have shown nonsocial stimuli to be more effective. For example, Darby and Riopelle found observational learning to be inferior to discrimination learning-set performance with nonsocial stimuli, even after considerable experience $(1,000$ problems $)$. The experiment in this report does not directly compare social and nonsocial stimuli, but it does investigate whether or not changes across successive reversals of social stimuli are similar to those that would be expected with nonsocial events.

\section{METHOD}

The Ss were two male squirrel monkeys (Saimiri sciureus) that were maintained at $80 \%$ of their free-feeding weights by food deprivation. One $S$ served as the stimulus monkey at all times and the other always as the $O$. The Ss were the same as those used in the previous experiments.

The apparatus consisted of two separate environments that acoustically separated the monkeys but allowed visual inspection between the chambers. The stimulus monkey's chamber contained a chain manipulandum that operated a microswitch, a trough into which 45-mg Noyes pellets were dispensed automatically, and pilot lamps with a shield that permitted stimuli to be presented to the stimulus monkey but not to the $O$ (Danson \& Creed, 1970).

The $O$ 's environment contained a lever-operated microswitch on the wall adjacent to the stimulus monkey's chamber, and a trough below the lever received $45-\mathrm{mg}$ Noyes pellets from an automatic feeder. The contingencies of the experiment were arranged on relay programming equipment situated in a room adjacent to the quarters that housed the experimental chambers. A more detailed description of the apparatus is found in the earlier report.

The stimulus monkey was trained on a schedule of reinforcement in which every seventh response (chain pull) in the presence of an illuminated red jewel pilot lamp (SD) produced a 45-mg Noyes pellet, while responses in the absence of the light $(S \Delta)$ were not reinforced (mult FR 7 Ext). The average $S^{D}$ duration was $13 / 4 \mathrm{~min}$, which varied between the limits of $1 \mathrm{~min}$ and $2 \frac{1}{2} \mathrm{~min}$. The SD was terminated with the first reinforcement after the variable duration had elapsed. The $\mathbf{S} \Delta$ duration was programmed on a separate variable interval schedule with an average of $2-2 / 3 \mathrm{~min}$, which varied between the limits of $1 \mathrm{~min}$ and $4-1 / 3 \mathrm{~min}$. The schedule resulted in a rate of chain pulling that averaged about 100 responses/min during $\mathrm{SD}^{\mathrm{D}}$ and approached 0 responses/min during $\mathrm{S} \Delta$.

The $O$ had been trained on a schedule of reinforcement in which every sixth response was reinforced in the presence of the stimulus monkey's chain pulling behavior, but no reinforcement could be obtained when the stimulus monkey was not chain pulling (mult FR 6 Ext). The $O$ had received approximately $40 \mathrm{~h}$ of training on this schedule before the start of the present experiment. Thus, the first reversal consisted of not responding of the stimulus monkey serving as the $S^{D}$ for the $O$ and the high rate of the stimulus monkey as $\mathrm{S}^{\Delta}$.

Table 1

Observer Monkey's Number of Sessions, Errors, and Reinforcements to Reach Criterion During Each Reversal

\begin{tabular}{|c|c|c|c|c|c|c|c|}
\hline \multirow[b]{2}{*}{ 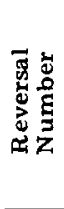 } & \multicolumn{3}{|c|}{ Zero Rate of Response as $S^{D}$} & \multicolumn{4}{|c|}{ High Rate of Response as SD } \\
\hline & $\begin{array}{l}\text { Number } \\
\text { of } \\
\text { Sessions } \\
\text { to } \\
\text { Criterion }\end{array}$ & $\begin{array}{c}\text { Number } \\
\text { of } \\
\text { Errors } \\
\text { to } \\
\text { Criterion }\end{array}$ & $\begin{array}{l}\text { Number of } \\
\text { Reinforce- } \\
\text { ments } \\
\text { to } \\
\text { Criterion }\end{array}$ & $\begin{array}{l}\text { Reversal } \\
\text { Number }\end{array}$ & $\begin{array}{l}\text { Number } \\
\text { of } \\
\text { Sessions } \\
\text { to } \\
\text { Criterion }\end{array}$ & $\begin{array}{c}\text { Number } \\
\text { of } \\
\text { Errors } \\
\text { to } \\
\text { Criterion }\end{array}$ & $\begin{array}{l}\text { Number of } \\
\text { Reinforce- } \\
\text { ments } \\
\text { to } \\
\text { Criterion }\end{array}$ \\
\hline 1 & 34 & 8582 & 936 & 2 & 8 & 2076 & 503 \\
\hline 3 & 11 & 2142 & 834 & 4 & 1 & 104 & 74 \\
\hline 5 & 2 & 228 & 138 & 6 & 2 & 456 & 158 \\
\hline
\end{tabular}




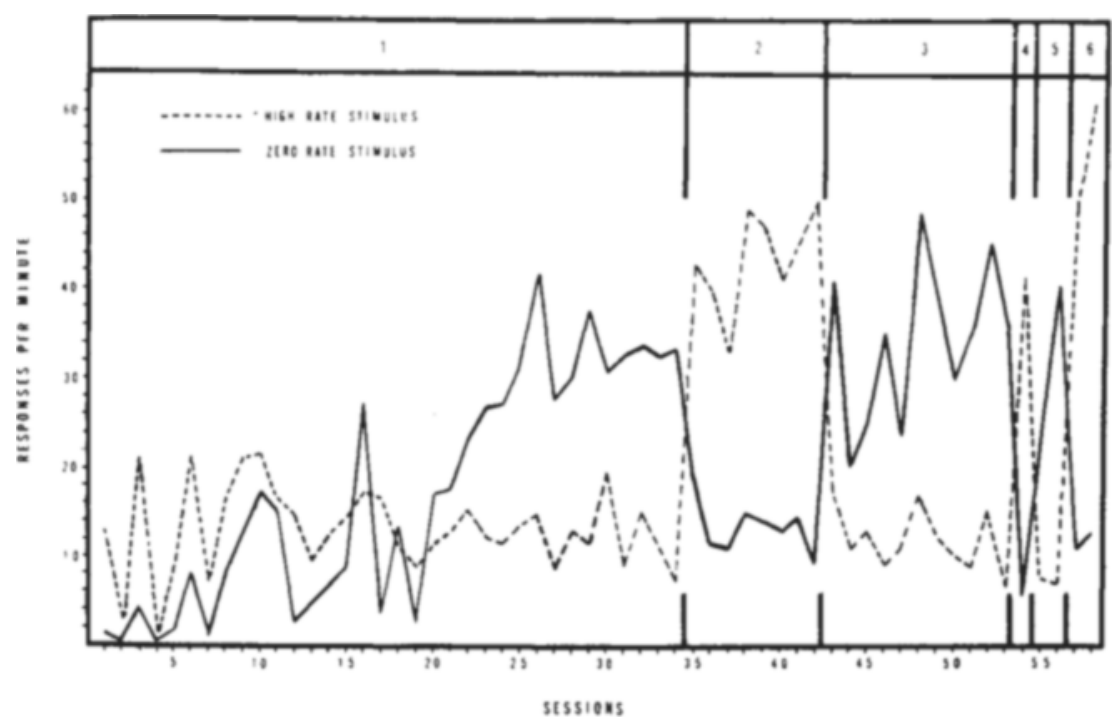

Fig. 1. Responses per minute of the observer monkey in the presence of the high rate of response (dotted line) and the near-zero rate (solid line) of the stimulus monkey. Numbers 1 through 6 at the top of the figure designate the consecutive reversals of the experiment and correspond to the numbers presented in Table 1 . The zero rate of the stimulus monkey served as $S^{D}$ during the odd-numbered segments and the high rate during the even-numbered sections.

The criterion used to initiate a reversal was that the $O$ 's rate during $\mathrm{SD}^{\mathrm{D}}$ was five times greater than his rate during $\mathrm{S}^{\Delta}$. On the session following the attainment of the criterion; the previous $\mathrm{S}^{\mathrm{D}}$ and $\mathrm{S}^{\Delta}$ and the duration that each stimulus condition remained in effect (see above) were reversed. Six successive reversals were carried out in this fashion. Each session was $30 \mathrm{~min}$ in duration, and six sessions were performed weekly, each at approximately the same time on successive days.

\section{RESULTS AND DISCUSSION}

The number of sessions to reach criterion, the number of responses that the $\mathrm{O}$ emitted in the presence of $\mathrm{S} \Delta$ (errors), and the number of reinforcements that the $\mathrm{O}$ obtained in reaching criterion is shown for each reversal in Table 1.

The table generally shows a reduction in sessions to criterion, errors, and reinforcements across successive reversals, both when the zero rate (left side of table) and the high rate of response (right side of table) served as $\mathrm{S}^{\mathrm{D}}$. In interpreting the results presented in the table, it should be noted that before the reversal study began, the $O$ had received extensive training under conditions in which the high rate of the stimulus monkey served as $\mathrm{SD}^{\mathrm{D}}$ and the zero rate as $\mathrm{S} \Delta$. The extended training may have affected the measures obtained during the first reversal (zero rate as $\mathrm{SD}$ ) and also influenced the intial difference in attaining the criterion between the two $\mathrm{SD}_{\mathrm{S}}$. Notwithstanding this restriction, the table clearly shows that the second and third reversals for each discrimination were acquired more rapidly than the first.

The rate of response of the $\mathrm{O}$ in the presence of the high and zero rate of response of the stimulus monkey is shown for each session of the experiment in Fig. 1. The figure shows that during the first reversal, the rate of response in $S^{\Delta}$ was greater than the rate in $S^{D}$ for the first 15 sessions. On each of the following reversals, however, the rate of response in the presence of $S^{D}$ was greater than during $\mathrm{S}^{\Delta}$ on the first day of the reversal and remained higher until the criterion of $5: 1$ was met; this result is in agreement with the changes in the criterion measures shown in Table 1.

The findings of this experiment extend the authors' earlier work in two respects: First, the fact that a $S^{D}$ for the performance of another organism in much the same manner as a high rate of performance indicates that a potentially wide range of rates of response of one organism may develop discriminative control of another individual; second, the fact that the acquisition of the reversals at the end of the sequence was more rapid than the development of the discriminations at the beginning of the series suggests that the learning-set phenomenon is applicable to social stimuli and, more generally, to social learning. While not receiving explicit reference or a great deal of experimental corroboration in a social near-zero rate of response may serve as context, the learning-set phenomenon has been implicated in social learning theory. For example, Gewirtz \& Stingle (1968) have presented the view that generalized imitation, the copying by an $O$ of many different behaviors of a model in diverse situations, is the result of the operant history of the organism (i.e., the history of the controlling discriminative and reinforcing stimuli). An organism learns to imitate novel responses because of a history of being reinforced for matching other of the model's behavior. The formation of a learning set, then, might be offered as a likely process by which novel behaviors come to be imitated. This analysis seems to be supported by the presented data, as well as by some experimentation with humans (Baer, Peterson, \& Sherman, 1967; Lovaas, Berberich, \& Perloff, 1966; Peterson, 1968 ), but, while the evidence makes a learning-set explanation attractive, the present evidence obviously does not represent a direct test of this contention.

Since a near-zero rate of the stimulus monkey gained discriminative control of the $\mathrm{O}$, the present findings might raise the question: Can a near-zero rate of response (not responding) serve as a social stimulus? Two proposals seem worthy of discussion in this regard: (1) The $O$ was under the control of some other behavior of the stimulus monkey, which occurred consistently when that $\mathrm{S}$ was not responding (chain pulling); (2) the $O$ was under the control of the time between performances or the time since the last performance of the stimulus monkey. Judging from the event records collected during the experiment, the latter possibility seems the more likely of the two to be correct. The records consistently showed that when the high rate of the stimulus monkey served as the $\mathrm{S}^{\mathrm{D}}$, the $O$ began barpressing as soon as chain pulling started, but when the zero rate served as $\mathrm{S}^{\mathbf{D}}$, there was consistently a long latency between the termination of chain pulling of the stimulus monkey and the first response of the $O$. If the $O$ had been under the control of some performance of the stimulus monkey other than chain pulling, then it seems unlikely that this other performance would occur only after chain pulling had ceased for some time. Unfortunately, the death of the $O$ shortly after this experiment prevented a further examination.

\section{REFERENCES}

BEAR, D. M., PETERSON, R. F., \& SHERMAN, J. A. The development of imitation by reinforcing behavioral similarity to a model. Journal of the Experimental Analysis of Behavior, 1967, $10,405-416$ 
DANSON, C., \& CREED, T. Rate of response as a visual social stimulus. Journal of the Experimental Analysis of Behavior, $1970,13,233-242$

DARBY, C. L., \& RIOPELLE, A. J. Observational learning in the rhesus monkey. Journal of Comparative \& Physiological Psychology, 1959, 52. 94-97.

GEWIRTZ，J. H., \& STINGLE, K. G. Learning of generalized imitation as the basis for identification. Psychological
Review, 1968, 75, 374-396.

KIMBLE G. A Hilgard \& Marquis Conditioning and learning. (Rev. ed.) New York: Appleton-Century-Crofts, 1961.

LOVAAS, O. I., BERBERICH, B. F., \& PERLOFF, B. F Acquisition of imitative speech by schizophrenic children. Science, 1966, 151, 705-707.

PETERSON, R. F. Some experiments on the organization of a class of imitative behaviors. Journal of Applied Behavior Analysis, 1968, 1, 225-235.

\section{Differential resistance to extinction as a function of fixed-interval contrast in training*}

\author{
MELVIN H. MARX and DAVID W. WITTER \\ University of Missouri, Columbia, Mo. 65201
}

Differential fixed-interval schedules were used to test the previously advanced motivational theorv of extinction, which predicts positive or negative contrast effects in extinction as a function of acquisition experience. Rats trained on both FI 10-sec and FI 40-sec schedules of bar presentation and extinguished on FI $10 \mathrm{sec}$ (all within a discrete-trial experimental design) were significantly more resistant to extinction than were control Ss both trained and extinguished on FI 10 sec. On the other hand, rats receiving mixed training and extinguished on FI $40 \mathrm{sec}$ were significantly less resistant to extinction than were control Ss trained and extinguished on FI $40 \mathrm{sec}$. These findings are interpreted as lending additional support to the motivational theory of extinction.

This experiment was performed to test a prediction derived from the motivational theory of extinction advanced earlier (Marx, 1966). A number of experiments were performed with animals whose training was administered under two contrasting conditions, one more "preferred" than the other (e.g., FR 1 and FR 5, 0-sec and 20 -sec delay in goalbox). These animals were then extinguished in either the preferred or the nonpreferred condition and compared in each case with controls that had been trained only under that condition. The rationale for tho prediction of superior performance for the animals tested under the preferred condition (a form of positive contrast) and inferior performance for those

*This research was supported in part by Grants GB5853 and GB8506 from the National Science Foundation and by Research Career Award 1-K-6-MH 22023 to the first author from the National Institute of Mental Health. tested under the nonpreferred condition (a form of negative contrast) was that the contrast with the other training condition diminished the effect of extinction in the first case and enhanced it in the second. The resulting alteration in the animal's motivation to make the instrumental response would then reflect either a positive contrast effect in extinction (PCEE) or a negative contrast effect in extinction (NCEE). This prediction was generally supported in the various experiments.

The present experiment extends this experimental design to training under fixed-interval reinforcement conditions, applied within a discrete-trial (controlled-operant) framework. An important advantage of the FI schedule for the present purpose is that it provides two especially sensitive measures of motivation-the number of instrumental responses emitted during the interval before reinforcement, as well as the latency of the first response. However, because of the deviation from orthodox Skinnerian usage, the meaning of the term "FI schedule" within the present experimental context must be clearly stated. This term here refers solely to the program of bar presentation and retraction and to the relationship of barpresses and consequent magazine operations to that program. In training, magazine operations produced by barpresses within the period of bar presentation provided food (reinforcement), whereas, in extinction, barpresses operated the magazine in exactly the same manner but produced no food (empty magazines). Thus, the training and extinction schedules of bar presentation and magazine operation were identical, the only difference being the absence of food in the latter phase.

In this experiment it was predicted that rats trained under both FI 40 -sec and FI $10-\mathrm{sec}$ conditions would extinguish more rapidly when tested on the less preferred FI 40-sec schedule, as compared with rats trained and tested only on that schedule. Similarly, such rats were predicted to extinguish more slowly when tested under the FI 10-sec conditions and compared with the appropriate controls (FI 10-sec training and testing).

\section{SUBJECTS}

The Ss were 40 young ( 3 months) and experimentally naive female hooded rats from the Long-Evans strain maintained by the Department of Psychology. Three Ss were lost during the course of the experiment from death or failure to train, leaving two groups with $10 \mathrm{Ss}$ each, one with 9 , and one with 8 .

\section{APPARATUS}

The controlled operant conditioning boxes used have been described in detail previously (Marx, Tombaugh, Hatch, \& Tombaugh, 1965). For this experiment they were operated by, and the data were recorded by, a Honeywell DDP-116 computer. Two retractable bars with associated food magazines were used in each of the eight boxes.

\section{EXPERIMENTAL DESIGN}

The design was a 2 by 2 factorial, with two discrete-trial training conditions (experimental Ss trained on both FI 10 -sec and FI 40-sec schedules, control Ss trained on either FI 10-sec or FI 40-sec schedules), and two extinction conditions (FI $10-\mathrm{sec}$ or FI 40-sec schedules).

\section{PROCEDURE}

The Ss were maintained for 1 week on a reduced feeding program to lower their body weights to $85 \%$ of the ad lib level. Thereafter, they were given a maximum of $10 \mathrm{~g}$ daily of 